\title{
Minimally invasive thoracic surgery for empyema
}

Cite as: Subotic D, Lardinois D, Hojski A. Minimally invasive thoracic surgery for empyema. Breathe 2018; 14: 302-310.
The widely accepted and still increasing use of video-assisted thoracic surgery (VATS) in pleuropulmonary pathology imposes the need to deal with two major pitfalls: the first is to avoid its unselective use, while the second relates to inappropriate rejection of VATS on the basis of "insufficient radicality". Unlike a quite established role of VATS in lung cancer patients, in patients with pleural empyema, the role of VATS is less clearly defined. The current evidence about VATS in patients with pleural empyema could be summarised as follows: VATS is accepted as a useful treatment option for fibrinopurulent empyema, but the treatment failure rate increases with the increasing proportion of stage III empyema, necessitating further surgical options like thoracotomy and decortication. As both pulmonologists and surgeons deal with diagnosis and treatment of pleural empyema, this article is an attempt to highlight the existing evidence in a more user-friendly way in order to help practising physicians to optimise the use of VATS in these patients. In other words, in the absence of randomised studies comparing VATS and thoracotomy, the key question to be answered is: are there any pre-operative findings that can be used to select patients for initial VATS versus proceeding directly to a thoracotomy?

@ERSpublications

Early treatment by video-assisted thoracic surgery improves the outcome of stage I and II, as well as of carefully selected stage III, pleural empyema; delay in surgical intervention is the most common predictor of conversion http://ow.ly/Sz6C30m64sL

\section{Pleural empyema as a medical and surgical problem}

The incidence of pleural empyema has continually increased since 1990. According to 2006 data, it affected over 65000 patients each year in the UK and USA [1]. Despite optimal medical management, it is still associated with significant morbidity and mortality.

The majority of indications for surgery in patients with pleural empyema relate to parapneumonic empyema. Most pleural effusions, occurring in around $40 \%$ of pneumonia patients, can be successfully treated conservatively by appropriate antibiotic treatment. However, around $10 \%$ of these effusions become loculated or progress to empyema [2]. In this case, a wide spectrum of therapeutic options is available, such as repeated thoracentesis with intrapleural antibiotic instillation, and chest tube drainage with or without intrapleural fibrinolytics and DNase. Drainage using videoassisted thoracic surgery (VATS) or open surgery are options as well [3]. It should be kept in mind that, after the Multicenter Intrapleural Sepsis Trial in 2005 [4], the routine use of fibrinolytics could not be supported by clear evidence; similarly, according to the Cochrane review of 2008, the 
benefit of fibrinolysis was not significant in highquality trials [5].

The comprehensive literature overview that would be helpful in everyday practice is complicated by inconsistency and imprecision in data reporting and by the current practice of dealing with this problem both by pulmonologists and surgeons. In order to avoid misleading conclusions, this aspect is addressed prior to discussing the possible treatment options.

\section{Time trends in the use of VATS in pleural empyema patients and points of confusion in data reporting}

Initially, VATS was used mostly for confirmation of the presence of empyema. Later, VATS debridement was found to be a very effective method of treating early fibrinopurulent empyema. More recently, VATS decortication has also been reported to successfully manage stage I/II empyema after failure of chest tube thoracostomy. Finally, its effectiveness in treating multiloculated and chronic empyemas has also been addressed [6].

One of the frequent causes of confusion in data reporting is overly general statements, such as "Several recent studies suggest that VATS and open thoracotomy have similar treatment success rates and are equally effective". Such a statement may be misleading unless the analysis was performed on well stage-matched groups, which is usually not the case. Even if better specified, statements like "minimally invasive (VATS) techniques offer a shorter duration of pleural drainage and hospital stay with a lower mortality and morbidity, compared with thoracotomy/decortication" should be accepted with caution. This is because VATS pleural "decortication" in these studies refer mainly to stage II empyema, without an organised pleural cortex. Moreover, lung re-expansion fails in as many as $41 \%$ of VATS cases of pleural debridement [7], so that these patients subsequently really required a thoracotomy and decortication.

In reports with the disease stage (stage II versus stage III) determined only by symptom duration ( $<3$ weeks versus $>3$ weeks), a statement like "VATS facilitates the management of fibrinopurulent or even organised pleural empyema" may at first sight seem appropriate. However, it is clear that the correct empyema stage assessment cannot be done without clear description of the radiographic aspect.

When reporting the difference in operating time between VATS and thoracotomy, the longer operating time in the thoracotomy group can be explained by the extra time during attempts to perform unsuccessful VATS debridement/decortication.

Conversely, in studies with upfront classification into thoracotomy and VATS groups, there is a real bias that a primary thoracotomy precludes knowing if a successful VATS might be performed in these patients. The reports from authors who always begin with VATS and change to thoracotomy if necessary are more reliable in terms of predictors of a successful operation [8].

Many series include in the analysis empyema forms other than parapneumonic, such as post-operative, tuberculous or post-traumatic empyema, thus making the comparison among studies less reliable.

An example of properly addressed methodological limitations is: "there was no correlation between length of pre-operative management and length of post-operative hospital stay". This statement was further explained by the information that $23 \%$ of the analysed patients were transferred back to their local hospital, while a further $25 \%$ were discharged home with a chest drain in situ [9].

\section{Some basic considerations: aetiology and clinical classification}

For clinical purposes, pleural empyemas can be divided into: 1) primary forms, from pulmonary infectious diseases (pneumonia, abscesses, tuberculosis, descending necrotising mediastinitis) or extra-thoracic ones (sub-phrenic abscesses, pancreatitis, intestinal perforations, peritonitis with pleura fistula); and 2) secondary forms due to iatrogenic causes, such as diagnostic and surgical procedures, traumas (pneumothorax, haemothorax) and tumours (advanced lung cancers, tracheobronchial fistulas, oesophageal fistulas, osteonecrosis).

Empyema can be differentiated into three phases, exudative (stage I), fibrinopurulent (stage II) and organising (stage III), representing a continuously evolving process that can be arrested by therapeutic intervention. Fibrinopurulent empyema changes into an organising phase within 7-10 days of symptom initiation. In addition, lung entrapment should be suspected when the pleural infection process is known to have been ongoing for longer than 10-14 days [10].

The acute or exudative stage (stage I) has been characterised by a thin serous fluid with minimal debris, $\mathrm{pH}>7.2$, lactate dehydrogenase $<1000 \mathrm{IU} \cdot \mathrm{L}^{-1}$, glucose $>60 \mathrm{mg} \cdot \mathrm{dL}^{-1}$, negative culture and no loculations. The fibrinopurulent stage (stage II) has been characterised by a thick fluid and thick fibrin strands, $\mathrm{pH}<7.2$, lactate dehydrogenase $>1000 \mathrm{IU} \cdot \mathrm{L}^{-1}$, glucose $<60 \mathrm{mg} \cdot \mathrm{dL}^{-1}$, positive culture or presence of suppuration, and increased loculations in the pleural cavity.

\section{Therapeutic approach}

The treatment rationale for pyogenic pleural empyema is: 1) control of ongoing infection; and 2) prevention of recurrent infection and subsequent late 
restriction. Despite progress in imaging techniques and therapeutic tools, a high proportion of stage III empyema still exists in many reported series, possibly due to prolonged hospitalisation and physicians' and pulmonologists' preference for conventional therapies, such as antibiotics and insertion of a pigtail catheter, over surgery. There is almost a consensus that this may cause late referral and further complications of the empyema cases. The attitude that late referral to surgery exposes the patient to devastating morbidity is also sufficiently evidence based [11].

Unlike the situation 15 years ago, where the main question related to the optimal time for open decortication, nowadays there is an additional question: when is the optimal time for VATS? The absence of clear guidelines for the use of VATS in pleural empyema influences the treatment outcome as well.

Independent of the pleural empyema stage, bronchoscopic exploration (even when computed tomography (CT) does not suggest any underlying lesion), aimed mainly to rule out malignancy and other endobronchial lesions, is mandatory because if malignancy or specific lesions are found, the therapeutic approach is different, as will be discussed in the section about VATS and tuberculous empyema.

\section{Early stage of pleural empyema}

In the exudative stage, closed chest drainage with appropriate antibiotics can be effective and such an approach is widely accepted. The main problem in practice is the inability to reliably determine the evolution towards stage II, with development of loculations and/or a visceral pleural cortex, when antibiotic treatment and tube drainage alone become ineffective for sepsis control and achieving full lung re-expansion. According to British Thoracic Society Pleural Disease Group published clinical guidelines, in the absence of clinical improvement of the sepsis at 5-7 days, patients considered fit for surgery should be referred for surgical treatment, either in form of VATS or open decortication [12]. Although the expert panel could not suggest objective criteria to define the point at which a patient should proceed to surgery, patients with purulent fluid and/or loculations at presentation or with residual sepsis syndrome and persistent pleural collection despite drainage and antibiotics are considered more likely to benefit from surgical debridement of the pleural cavity. However, no recommendation was given on the choice of surgical approach: VATS, open thoracic drainage or thoracotomy. An example of VATS surgery in pleural empyema stage I is presented in figure 1 .

Stage II empyema is a transitory stage between the exudative (stage I) and chronic (stage III) empyema, representing only a short time frame in the evolution towards chronicity. At this time point, VATS techniques can achieve pleural cavity clearance and lung re-expansion through pulmonary surface debridement and breakdown of loculations [13]. It is important to point out that the appropriate VATS intervention at this stage
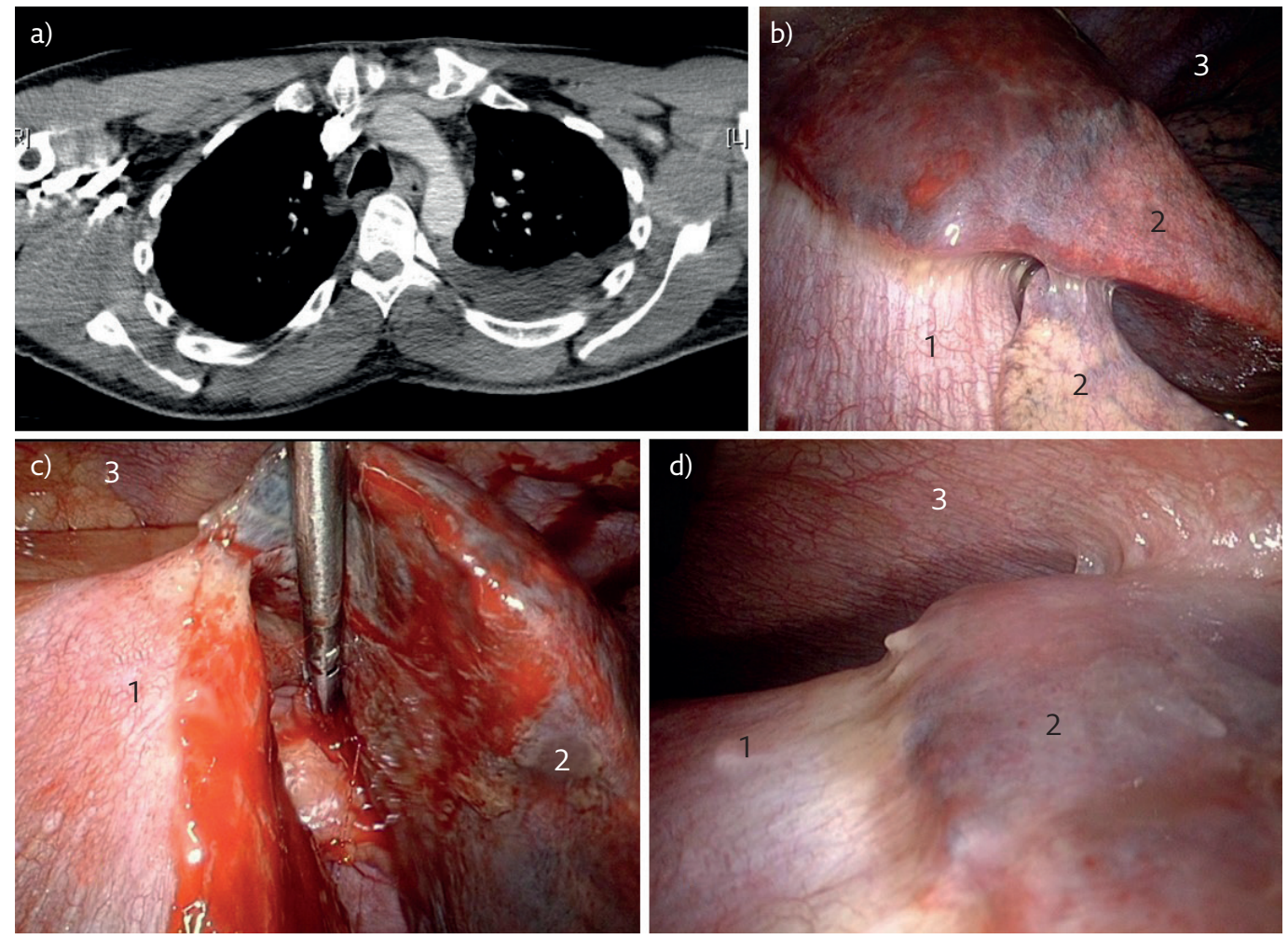

Figure 1 VATS debridement in pleural empyema stage I. a) Pre-operative CT of the thorax; $b$-d) operative views. 1: diaphragm; 2: lung; 3: chest wall. 
comprises thorough lung liberation with removal of the peel not only from the visceral pleura, but also with complete debridement of the parietal pleura, costo-diaphragmal and costo-mediastinal recesses as well. Such a procedure is not equal to the so-called "medical thoracoscopy", aimed mostly to determine the empyema stage, rule out specific pleuritis or pleural carcinosis and eventually to break down loculations and adhesions before the chest tube insertion. An example of VATS decortication for pleural empyema stage II is presented in figure 2 .

Early VATS drainage or decortication has been reported to offer better early results than treatment with fibrinolytics or thoracostomy alone [14]. The current British Thoracic Society guidelines suggest consideration of surgical treatment in patients with persisting sepsis and pleural collection, with a maximum period of 7 days without resolution [12].

LARDINOIS et al. [15] clearly demonstrated that the probability of conversion to thoracotomy for fibrinopurulent empyema increases from $22 \%$ to $86 \%$ between day 12 and day 16 of presentation. Chung et al. [16], in their recent retrospective analysis of 120 cases of VATS empyemectomies, confirmed the importance of early referral to surgery, demonstrating that patients with a symptom duration of $<4$ weeks had better early results compared with a symptom duration $>4$ weeks.

To date, there have only been two randomised controlled trials comparing VATS and tube thoracostomy as the primary intervention [17, 18]. Both reported that patients undergoing VATS as the primary management had fewer treatment failures and shorter length of hospital stay. The sample groups involved were relatively small $(n=104$ and $n=20$ ). The focus of the trial by WozNIAK et al. [17] was the success of the first intervention, success being defined as no death and no additional drainage procedures. Drainage or pigtail catheter were shown to be less successful than operative procedures (success rates: pigtail 40\%, drainage $38 \%$, VATS $81 \%$, thoracotomy $89 \%$ ). The overall mortality was $17 \%$ and was unaffected by empyema stage. Cause-specific death was $15 \%$ for primary (parapneumonic) and $21 \%$ for secondary (postoperative, trauma) empyema. Major complications occurred in $78 \%$ of patients in whom the first procedure failed and in $18 \%$ of patients with successful first procedure (detailed morbidity was not reported). Importantly, the strongest predictor of treatment failure and mortality was drainage as the first procedure. In the trial by WAIT et al. [18] (nine patients undergoing drainage plus streptokinase, 11 undergoing VATS), each group suffered one mortality (not significant), morbidity not being specified. The VATS group had a significantly higher primary treatment success ( $91 \%$ versus $44 \%$ ), lower chest tube duration ( $5.8 \pm 1.1$ versus $9.8 \pm 1.3$ days) and lower number of total hospital days (8.7 \pm 0.9 versus $12.8 \pm 1.1$ days).

In brief, the existing evidence suggests that aggressive, early treatment including VATS improves the outcome of stage I-II pleural empyema. These studies have led some researchers to advocate a paradigm shift in empyema treatment and propose VATS as the treatment of choice at diagnosis for all patients with fibrinopurulent empyema [19]. However, international guidelines recognise a definite role for VATS only after failure of conservative treatment.

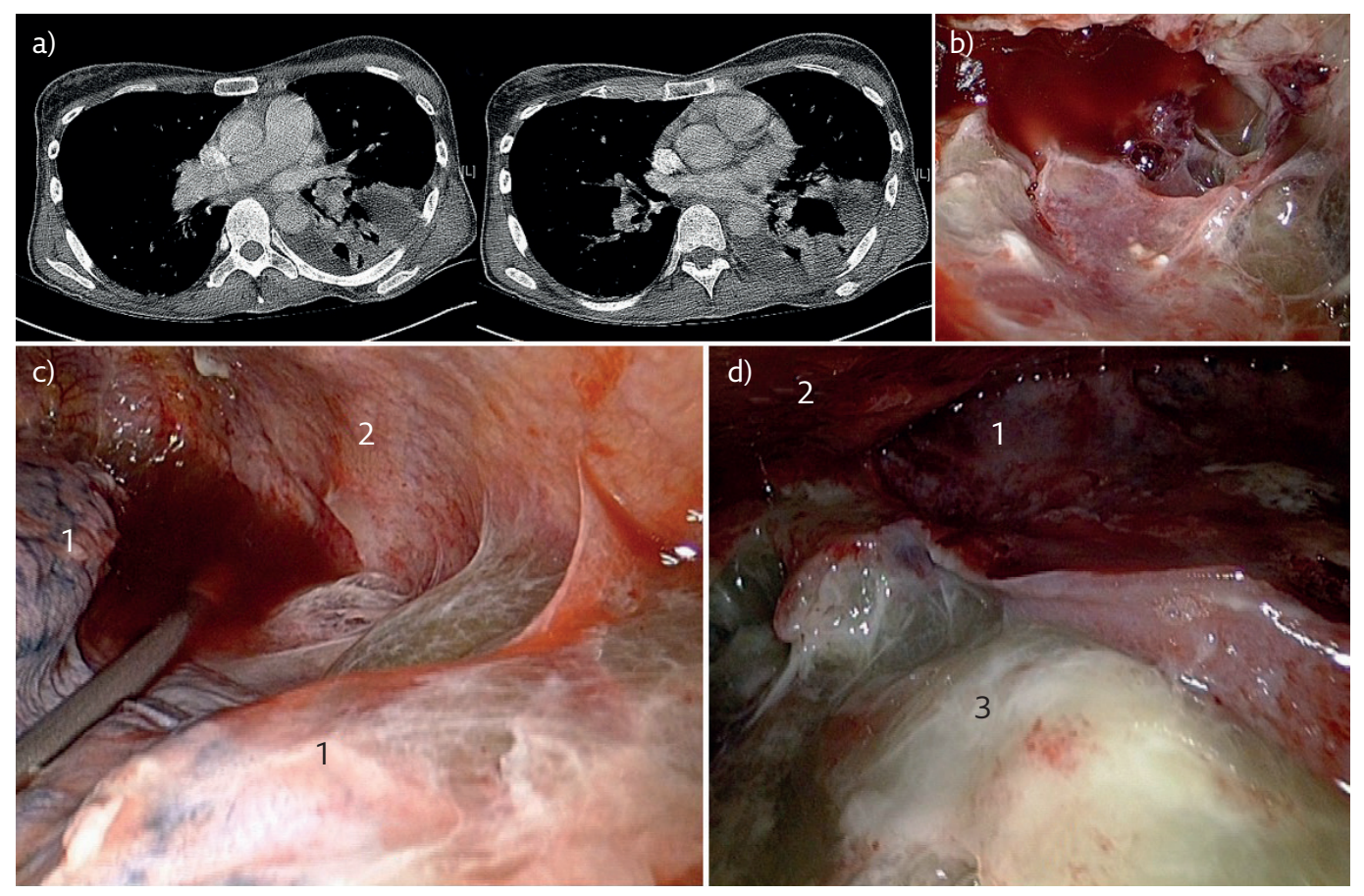

Figure 2 VATS decortication in pleural empyema stage II. a) Pre-operative CT of the thorax; $b$-d) operative views with multiple intrapleural loculations. 1: lung; 2: chest wall; 3: sub-pulmonary fibrin collection. 


\section{Late stage of empyema}

In stage III pleural empyema, the insertions of the empyema sac, extending frequently deep in the mediastinum, are in close contact with important structures like the oesophagus, superior vena cava and aorta, making a decortication not a trivial operation.

Although the evidence about optimal timing for surgery in this empyema stage is lacking, the need for surgical treatment is not in debate. Bearing in mind that delays in performing surgical intervention lead to deterioration of patient status and a worse post-operative patient condition, the importance of optimal timing for surgery in earlier stages, in order to prevent stage III occurrence, clearly overweighs the considerations of the roles of VATS and open surgery as first-line treatments.

The choice of appropriate treatment is still difficult, owing to the absence of specific clinical, radiological and laboratory criteria for appropriate pre-operative staging of empyema. Furthermore, there are no clear guidelines for stage III pleural empyema and the nature of the underlying disease and the patient's overall condition must be taken into consideration as well [20]. The 2015 European Association for Cardio-Thoracic Surgery (EACTS) guidelines favoured VATS in patients with stage IIIII pleural empyema, although this approach was considered controversial, especially in patients with stage III empyema with a long ( $>5$ weeks) symptomatic clinical history [21].

The limitations of VATS at this stage and the reasons for conversion to thoracotomy are the inability to access the cavity with the thoracoscope because of strong adhesions between parietal and visceral pleura or the inability to obtain an adequate pleural decortication to achieve lung re-expansion [19, 22]. Potential contraindications and drawbacks of VATS include the inability to tolerate single lung ventilation, severe coagulopathy and operative time with increased costs. In addition, a minimally invasive approach requires a significant learning curve with a high risk of conversion rate, up to $86 \%$ [23].

In clinical practice, it is difficult to identify when an advanced stage disease will need a true decortication or blunt stripping of the pleural peel, which can be easily performed by VATS. This evaluation is usually performed at the time of operation [8].

The existing evidence justifies both frequently used approaches in late-stage pleural empyema. In the first approach, in patients with a longlasting history, a thickened pleural peel and signs of restriction on CT scan, and those with CT scan signs of an abscess or a tumour, a primary thoracotomy and decortication is advocated. In all other situations, the patients are informed that a VATS approach will be attempted, and informed consent is obtained to proceed with thoracotomy
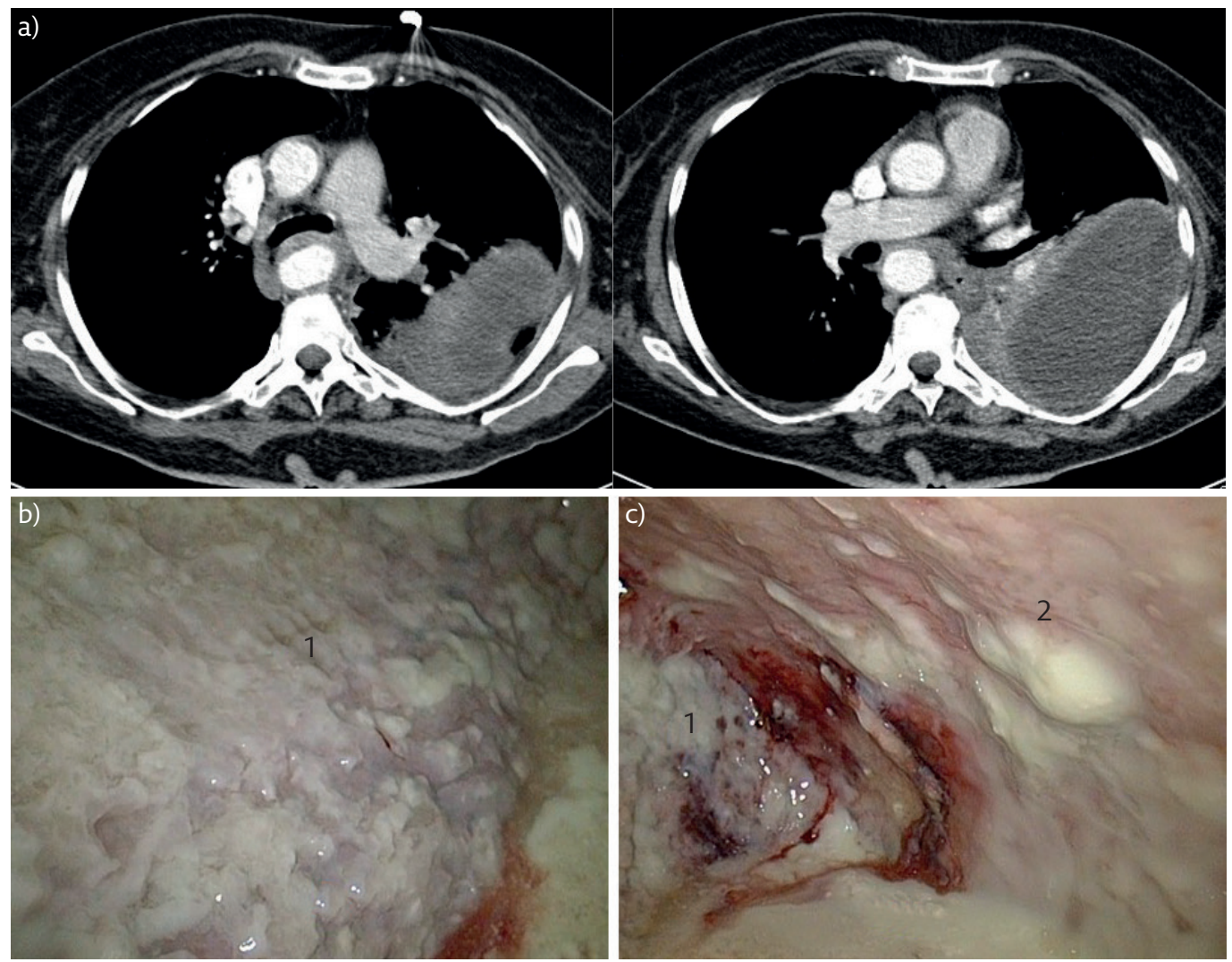

Figure 3 VATS decortication in pleural empyema stage III. a) Pre-operative CT of the thorax; $b$ and c) operative views with thick fibrin layer over the parietal pleura and cortex overlying the lung. 1: lung; 2: chest wall. 
in case of VATS failure [15]. The second approach, despite the high (4-40\%) reported conversion rates, favours VATS, performed by an experienced surgeon, as a first-choice treatment, instead of going directly to open decortication [16].

The key point in all stage III pleural empyema considerations can be summarised as follows: although some recent studies have demonstrated that VATS decortication can effectively manage stage III empyema, many authors still emphasise the importance of ensuring that the stage of empyema treated by each intervention is comparable, before suggesting that VATS is equivalent to the open procedure [24]. An example of the local aspect during VATS and open surgery for stage III pleural empyema is presented in figure 3 .

\section{What is the current clinical practice and can both therapeutic approaches be appropriate?}

In most clinical situations, the decision about surgical intervention is based on the patient's clinical status (for example fever, leukocytosis, chest radiography and chest $(T)$, and mainly relies on the physician's clinical experience, subjective opinion and available equipment. The reason why both of the aforementioned therapeutic approaches may be justified is the frequent finding that, despite a high conversion rate, the proportion of successful treatment outcomes of surgical treatment can be very high, in some series reaching 98\% [25].

An explanation for the diversity in practice is the fact that the pathomorphology of the stage III pleural empyema is not the same in all patients. In some patients, the main problem is the "plan de clivage" (cleavage plane) between the lung and the overlying peel; in others, the quality of the lung is a problem, or there are difficulties in reaching the peel insertion deep in the peri-diaphragmatic region or in the mediastinum. In these situations, the possibility of combining the advantages of VATS with mini- or limited thoracotomy, especially in high-risk patients, seems reasonable. One of the most important advantages of VATS in this setting is the ability to assess the lung re-expansion, thus allowing the surgeon to decide whether to proceed with further endoscopic manoeuvres or with a more extensive open approach [22].

One additional factor can improve the final treatment outcome of both techniques: the spontaneous fibrinolysis of the organism, taking place after both VATS and open decortication, as nicely demonstrated by Kно et al. [26]. As presented in figure 4, after VATS debridement alone, the initial size of the cavity decreased to $\sim 60 \%$ of the initial cavity volume. The fibrinolysis supported this effect by additionally decreasing the cavity dimensions to $\sim 30 \%$ of the initial size during the first 40 post-operative days. After open decortication, the initial surgery reduced the cavity to $\sim 42 \%$ of the initial size, with additional decrease by fibrinolysis to $11 \%$ of the initial size after 100 post-operative days. Owing to this physiological mechanism, the radiographic aspect of the operated patients at outpatient controls may be better than expected.

\section{Conversion rate, operative morbidity and mortality}

Conversion rates from VATS to thoracotomy range from $5.6 \%$ to $61 \%[8,27]$. The policy to attempt VATS first in every patient may partly explain the highest rates. As already mentioned, delay in surgical intervention has been shown to be the most common predictor of conversion. Unfortunately, the work of LARDINOIS et al. [15] is the only study that has specifically addressed this issue. Similar conclusions were obtained in the study by STEFANI et al. [25], who demonstrated that the probability of thoracotomy increased from $28 \%$ if the operation was performed within 10 days of the onset of symptoms to $81 \%$ if it was performed with a delay of 30-40 days.

Based on the existing evidence, radiological features do not seem to be a reliable predictor of conversion. In one study, it was found that only patients diagnosed with "empyema" by the radiologist were more likely to require a thoracotomy [8]. Conversely, two other studies concluded that the CT scan does not enable the selection of patients for successful VATS [22, 28]. However, we believe that the predictive value of

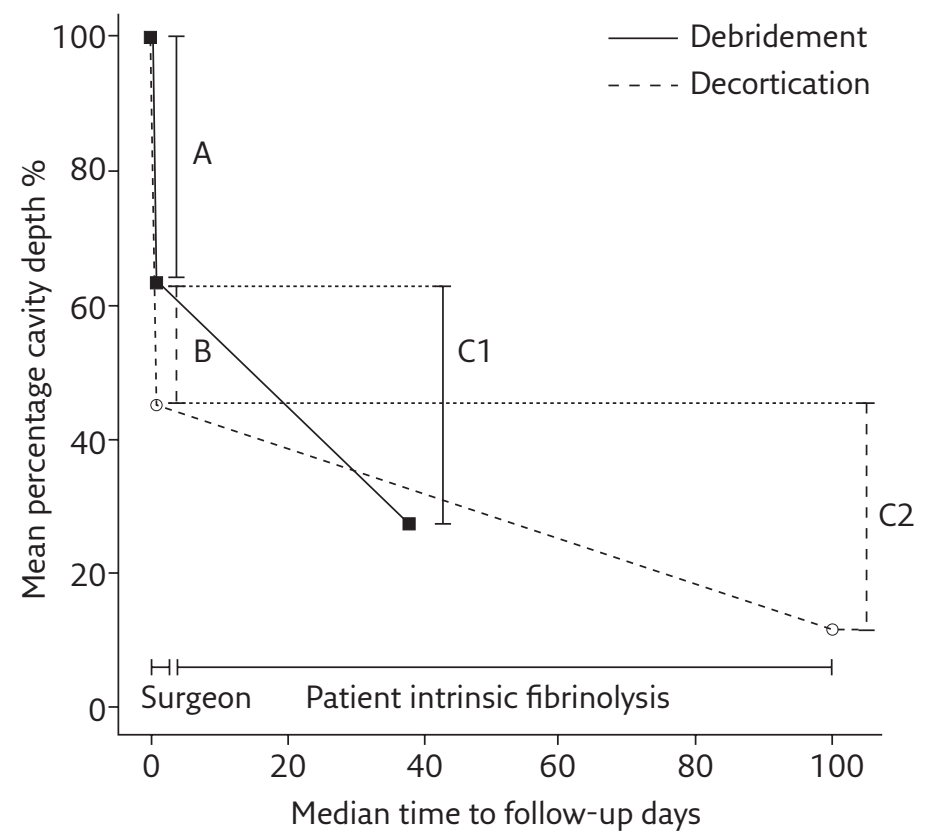

Figure 4 Effect of fibrinolysis on the empyema cavity size after VATS and open surgery. A: initial cavity size reduction by VATS debridement alone; $B$ : initial cavity size reduction by open decortication; C1: additional post-operative cavity size reduction by fibrinolysis during the first 40 post-operative days after VATS debridement; C2: additional effect of fibrinolysis during 100 post-operative days after the open surgery. Reproduced and modified from [26] with permission. 
$\mathrm{CT}$ is probably underreported, because most of the main features of stages II and III pleural empyema (major adhesions, loculations, fibrothorax with diffuse lung entrapment) can be reliably assessed before surgery.

Concerning pleural fluid microbiology as conversion predictor, data are conflicting. Positive microbiological cultures from pleural space are reported in $10-60 \%$ of patients, probably due to antibiotic treatment prior to sample collection. The sterility of the pleural fluid may also suggest that the continual presence of bacteria is not necessary to sustain the ongoing inflammatory response after the initial bacterial invasion [29]. Some studies have demonstrated that only Gram-negative bacteria significantly increased the rate of conversion [15], while in other studies, positive cultures had no influence on the conversion rate [25]. Such a finding can be explained by the systemic toxicity that prevents a monocyte-mediated fibroblast proliferation and a pleural cortex formation, in order to isolate the insulting bacterial infection. The absence of this mature pleural cortex makes surgery difficult, with significant bleeding as the plane of dissection between the cortex and visceral pleura is ill defined [30]. This can result in significant air leaks, bronchopleural fistulas and persistent pleural infection.

The reported complication rate after VATS decortication varies from $9 \%$ to $40.2 \%$ [11], the most frequent complications being prolonged air leak, bleeding, recurrence or persistence of the disease, surgical wound infection and residual pleural space. The 30-day post-operative mortality ranges from $1.3 \%$ to $6.6 \%$ [15].

\section{VATS and tuberculous empyema}

Most pleural effusions in patients diagnosed with tuberculosis respond well to anti-tuberculosis treatment, not progressing into empyema and thus not requiring further investigation in the absence of clinical signs of empyema/sepsis.

The problem that occurs in reports about tuberculous empyema is its inconsistent definition. In some reports the diagnosis is based 1) on the presence of acid-fast (AF) bacilli in the fluid or after culture of the effusion or 2) on the pleural biopsy. The second diagnostic option includes 1) pleural fluid smear (positive) for AF bacilli or 2) sputum (positive) for AF bacilli and radiographic lesions consistent with active parenchymal tuberculosis on radiography/CT (it is rare to have both). The third option is to accept the diagnosis of specific empyema only if Mycobacterium tuberculosis was isolated from pus and/or tissue.

Pleural fluid positivity varies from $20 \%$ to $71.6 \%$, reaching $93 \%$ in regions with high tuberculosis incidence [31]. Frequent culture negativity despite positive smears for $M$. tuberculosis in empyema fluid is attributed to the acidic, anaerobic environment of the pleural fluid and the lack of laboratory conditions required for mycobacterial culture, especially from purulent fluids [32]. Obtaining cultures from empyema fluid for M. tuberculosis is extremely important, bearing in mind a tendency to develop resistant organisms, either because antituberculosis drugs may not reach normal levels (due to thick pleura) or through poor patient compliance.

VATS decortication has also been reported to successfully manage tuberculous empyema [33, 34]. VATS seems to be a safe and accurate procedure to obtain a satisfactory toilet, as reported by CHEN et al. [35] in a study involving 274 patients with empyema secondary to tuberculosis. These authors noted an early recurrence or relapse (elevated temperatures between $38.5^{\circ} \mathrm{C}$ and $39.5^{\circ} \mathrm{C}$ and re-effusion with fibreboard) in three patients (1.1\%) and a late one in six patients $(2.3 \%)$ (re-effusion and surgical site infection after 6 months of intervention).

Interestingly, in some series, no tuberculous empyema was reported in stage II patients and it was detected only in $13.5 \%$ of stage III empyema patients despite the initial negative AF bacilli stain [23]. This can be the case especially in patients with completely obliterated pleural space, in whom the diagnosis of tuberculosis cannot be obtained until the pathohistological analysis of the operative specimen is complete (figure 5 ). In such situation, VATS is not suitable as the initial therapeutic step.

\section{VATS decortication in awake patients}

One particular advantage of a VATS approach is that it does not necessarily require a general anaesthesia. This is of particular importance in unstable patients with multiple comorbidities or in patients allergic to general anaesthesia. Rare reports have demonstrated that VATS decortication could effectively manage empyema in awake patients using epidural or paravertebral nerve block [36, 37]. It was even suggested that spontaneous lung ventilation resulted in easier dissection during the operation, resulting in lower post-operative morbidity.

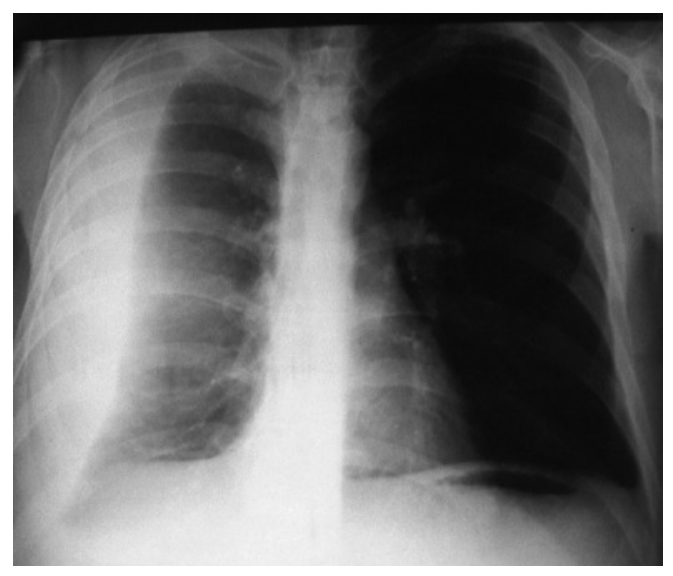

Figure 5 Late-stage tuberculous empyema. 


\section{Key points}

- Although early treatment by VATS improves the outcome of stage I-II pleural empyema, the international guidelines recognise a definite role for VATS only after failure of conservative treatment.

- There are no clear guidelines for stage III pleural empyema. The 2015 EACTS guidelines favoured VATS in patients with stage II-III pleural empyema, although this approach was considered controversial, especially in patients with stage III empyema with a long ( $>5$ weeks) symptomatic clinical history.

- VATS for pleural empyema should be performed in centres with experience in VATS and empyema surgery. We suggest a low threshold for conversion to thoracotomy in order to avoid unnecessary extending of the operation time and complications.

- $\quad$ CT diagnosis is crucial in the pre-operative decision-making process, giving the possibility of underlying disease assessment and localisation of loculations.

- From a technical stand-point, the thorough liberation both of the lung and all parts of the parietal pleura (costal, mediastinal and diaphragmal) is of utmost importance for the long-term outcome.

- Independent of empyema stage, delay in surgical intervention has been shown to be the most common predictor of conversion from VATS to thoracotomy.

\section{Conflict of interest}

None declared.

\section{References}

1. Ahmed RA, Marrie TJ, Huang JQ. Thoracic empyema in patients with community-acquired pneumonia. Am J Med 2006; 119: 877-883.

2. Light RW. Management of parapneumonic effusions. Arch Intern Med 1981; 141: 1339-1341.

3. Zahid I, Nagendran M, Routledge T, et al. Comparison of video-assisted thoracoscopic surgery and open surgery in the management of primary empyema. Curr Opin Pulm Med 2011; 17: 255-259

4. Maskell NA, Davies CW, Nunn AJ, et al. UK controlled trial of intrapleural streptokinase for pleural infection. N Engl J Med 2005; 352: 865-874.

5. Cameron R, Davies HR. Intra-pleural fibrinolytic therapy versus conservative management in the treatment of adult parapneumonic effusions and empyema. Cochrane Database Syst Rev 2008; 2: CD002312.

6. Drain AJ, Ferguson JI, Sayeed R, et al. Definitive management of advanced empyema by two-window video-assisted surgery. Asian Cardiovasc Thorac Ann 2007; 15: 238-239.

7. Waller DA, Rengarajan A. Thoracoscopic decortication: a role for video-assisted surgery in chronic postpneumonic pleural empyema. Ann Thorac Surg 2001; 71: 1813-1816.

8. Roberts JR. Minimally invasive surgery in the treatment of empyema: intraoperative decision making. Ann Thorac Surg 2003; 76: 225-230.

9. Wilson $\mathrm{H}$, Mohite P, Hall A, et al. Timing and efficacy of VATS debridement in the treatment of parapneumonic empyema. Arch Pulmonol Respir Care 2016; 2: 016-019.

10. Lee RB. Empyema thoracis. In: Sellke FW, del Nido PJ, Swanson SJ, eds. Sabiston and Spencer Surgery of the Chest. 7th Edn. Philadelphia, Elsevier Saunders, 2004; pp. 431-434.

11. Jagelavicius Z, Jovaisas V, Mataciunas $M$, et al. Preoperative predictors of conversion in thoracoscopic surgery for pleural empyema. Eur J Cardiothorac Surg 2017; 52: 70-75

12. Davies CW, Gleeson FV, Davies RJ. BTS guidelines for the management of pleural infection. Thorax 2003; 58: Suppl. 2, ii18-ii28.

13. Shahin Y, Duffy J, Beggs D, et al. Surgical management of primary empyema of the pleural cavity: outcome of 81 patients. Interact Cardiovasc Thorac Surg 2010; 10: 565-567

14. Petrakis IE, Kogerakis NE, Drositis IE, et al. Videoassisted thoracoscopic surgery for thoracic empyema: primarily, or after fibrinolytic therapy failure? Am J Surg 2004; 187: 471-474

15. Lardinois D, Gock M, Pezzetta E, et al. Delayed referral and Gram-negative organisms increase the conversion thoracotomy rate in patients undergoing video-assisted thoracoscopic surgery for empyema. Ann Thorac Surg 2005; 79: 1851-1856.

16. Chung $\mathrm{JH}$, Lee $\mathrm{SH}$, Kim KT, et al. Optimal timing of thoracoscopic drainage and decortication for empyema. Ann Thorac Surg 2014; 97: 224-229.

17. Wozniak CJ, Paull DE, Moezzi JE, et al. Choice of first intervention is related to outcomes in the management of empyema. Ann Thorac Surg 2009; 87: 1525-1530.

18. Wait MA, Sharma S, Hohn J, et al. A randomized trial of empyema therapy. Chest 1997; 111: 1548-1551.

19. Solaini L, Prusciano F, Bagioni P. Video-assisted thoracic surgery in the treatment of pleural empyema. Surg Endosc 2007; 21: 280-284

20. Weissberg D, Refaely Y. Pleural empyema: 24-year experience. Ann Thorac Surg 1996; 62: 1026-1029.

21. Scarci M, Abah U, Solli P, et al. EACTS expert consensus statement for surgical management of pleural empyema. Eur J Cardiothorac Surg 2015; 48: 642-653. 
22. Cassina PC, Hauser M, Hillejan L, et al. Video-assisted thoracoscopy in the treatment of pleural empyema: stagebased management and outcome. J Thorac Cardiovasc Surg 1999; 117: 234-238.

23. Divisi D, Gabriele F, Barone M, et al. Clinical history and surgical management of parapneumonic empyema what is the role of video-assisted thoracoscopic surgery (VATS)? Videoassist Thorac Surg 2017; 2: 65.

24. Edwin F, Frimpong-Boateng K. Is video-assisted thoracoscopic surgery really superior to open decortication for empyema thoracis? Interact Cardiovasc Thorac Surg 2009; 9: 78.

25. Stefani A, Aramini B, della Casa G, et al. Preoperative predictors of successful surgical treatment in the management of parapneumonic empyema. Ann Thorac Surg 2013; 96: 1812-1819.

26. Kho P, Karunanantham J, Leung M, et al. Debridement alone without decortication can achieve lung re-expansion in patients with empyema: an observational study. Interact Cardiovasc Thorac Surg 2011; 12: 724-727.

27. Landreneau RJ, Keenan RJ, Hazelrigg SR, et al. Thoracoscopy for empyema and hemothorax. Chest 1996; 109: 18-24.

28. Striffeler H, Gugger M, Im Hof V, et al. Video-assisted thoracoscopic surgery for fibrinopurulent pleural empyema in 67 patients. Ann Thorac Surg 1998; 65: 319-323.

29. Brims FJ, Lansley SM, Waterer GW, et al. Empyema thoracis: new insights into an old disease. Eur Respir Rev 2010; 19: $220-228$
30. Mandal AK, Thadepalli H, Mandal AK, et al. Outcome of primary empyema thoracis: therapeutic and microbiologic aspects. Ann Thorac Surg 1998; 66: 1782-1786.

31. Kundu S, Mitra S, Mukherjee S, et al. Adult thoracic empyema: a comparative analysis of tuberculous and nontuberculous etiology in 75 patients. Lung India 2010; 27: 196-201.

32. Nolte FS, Metchock B. Mycobacteria. In: Murray PR, Baron EJ, Pfaller MA, et al., eds. Manual of Clinical Microbiology. 6th Edn. Washington, American Society for Microbiology, 1995; pp. 400-437.

33. Barbetakis N, Paliouras D, Asteriou C, et al. The role of videoassisted thoracoscopic surgery in the management of tuberculous empyemas. Interact Cardiovasc Thorac Surg 2009; 8: 337-338.

34. Olgac G, Yilmaz MA, Ortakoylu MG, et al. Decisionmaking for lung resection in patients with empyema and collapsed lung due to tuberculosis. J Thorac Cardiovasc Surg 2005; 130: 131-135

35. Chen B, Zhang J, Ye Z, et al. Outcomes of videoassisted thoracic surgical decortication in 274 patients with tuberculous empyema. Ann Thorac Cardiovasc Surg 2015; 21 : 223-228.

36. Tacconi F, Pompeo E, Fabbi E, et al. Awake videoassisted pleural decortication for empyema thoracis. Eur J Cardiothorac Surg 2010; 37: 594-601.

37. Katlic MR. Video-assisted thoracic surgery utilizing local anesthesia and sedation. Eur J Cardiothorac Surg 2006; 30: 529-532. 